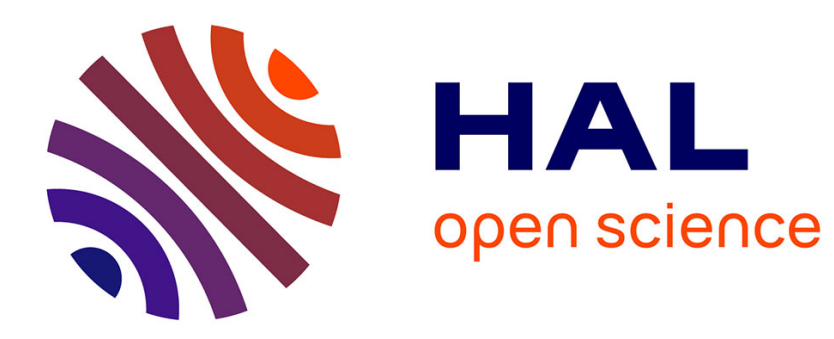

\title{
Parenté, noblesse et échec de la genèse de l'Etat: le cas allemand
}

\author{
Julien Demade
}

\section{To cite this version:}

Julien Demade. Parenté, noblesse et échec de la genèse de l'Etat: le cas allemand. Annales. Histoire, Sciences sociales, 2006, 61 (3), pp.609-631. halshs-00103662

\section{HAL Id: halshs-00103662 \\ https://shs.hal.science/halshs-00103662}

Submitted on 4 Oct 2006

HAL is a multi-disciplinary open access archive for the deposit and dissemination of scientific research documents, whether they are published or not. The documents may come from teaching and research institutions in France or abroad, or from public or private research centers.
L'archive ouverte pluridisciplinaire HAL, est destinée au dépôt et à la diffusion de documents scientifiques de niveau recherche, publiés ou non, émanant des établissements d'enseignement et de recherche français ou étrangers, des laboratoires publics ou privés. 


\section{Parenté, noblesse et échec de la genèse de l'État \\ Le cas allemand}

Julien Demade

L'entreprise de Joseph Morsel tient en l'analyse non pas de ce qui, classiquement, est perçu comme une défense victorieuse de la noblesse face à la construction d'un État lui enlevant ses prérogatives, mais de ce qu'il comprend comme la construction du pouvoir des nobles non titrés qui, entre $\mathrm{XIII}^{\mathrm{e}}$ et milieu du $\mathrm{XVI}^{\mathrm{e}}$ siècle, passent du statut de dépendants ministériaux à celui de "chevaliers immédiats d'Empire », au moment même où essaient de se bâtir des structures étatiques - et contre elles. Son enquête est donc caractérisée par un double renversement de la problématique : l'un, déterminé par le choix géographique, est le passage de l'étude de la genèse de l'État à celle de son échec ${ }^{1}$; l'autre est l'abandon du discours sur la « crise de la noblesse » à la fin du Moyen $\hat{A g e}^{2}$, vision historiographique paradoxalement aussi développée qu'ailleurs pour ces régions qui ont pourtant vu les vassaux résister avec succès à leur transformation en sujets.

L'enquête se concentre sur un groupe identifié par un même patronyme (un « lignage »), celui des Thüngen (sis en Basse-Franconie, dans l'évêché de Wurtzbourg). Cependant, « il ne s'agit pas de l'histoire des Thüngen, mais de l'histoire de l'espace social des Thüngen. Ainsi pris, les Thüngen se constituent en un observatoire sur les réseaux auxquels ils appartiennent $»$ : le choix d'un lignage n'est donc qu'une méthode permettant de constituer

\footnotetext{
${ }^{1}$ À propos de Joseph Morsel, La noblesse contre le prince : l'espace social des Thüngen à la fin du Moyen Âge (Franconie, v. 1250-1525), Stuttgart, Jan Thorbecke, 2000. Je remercie Christophe Duhamelle et Alain Guerreau pour leur relecture critique de ce texte, ainsi que Jacques Poloni-Simard pour son aide et sa patience extrême lors de son établissement définitif.
}

- Modalité qu'un lecteur français aurait tort de considérer comme une curiosité exotique sans grande importance au niveau global : rappelons que les régions concernées par ce phénomène sont l'Oberrhein, la Souabe et la Franconie, soit la plus grande partie de la moitié sud de l'espace allemand.

2 - Joseph Morsel, «Crise ? Quelle crise ? Remarques à propos de la prétendue crise de la noblesse allemande à la fin du Moyen Âge », Sources. Travaux historiques, 14, 1988, pp. 17-42. 
l'objet de recherche de façon cohérente, afin d'aboutir à un corpus clos rendant possible une mesure. Si l'histoire lignagère est un genre familier de l'historiographie médiéviste, ce n'est nullement d'elle qu'il s'agit ici, malgré l'identité apparente de l'objet, mais bien d'une étude de l'ensemble du groupe aristocratique, menée moins dans une perspective micro-historique qu'à travers les jeux d'échelles ${ }^{3}$.

Le résultat en est un remarquable renouvellement des catégories d'analyse (grâce à leur construction soigneuse, permettant d'éviter les pièges du sens commun historiographique), appuyé sur des méthodes trop peu répandues dans ce type de recherche (particulièrement la lexicométrie, plus généralement l'analyse quantitative), et l'intégration, en une structure explicative forte, d'un ensemble de problèmes généralement étudiés de façon disjointe ${ }^{4}$. Le propos est complexe; j'essaierai d'en retracer les grandes lignes, parfois en posant des accents différents, plus rarement en indiquant des désaccords.

\section{Transformation des structures et des fonctions de la parenté nobiliaire}

La première catégorie à déconstruire, ou plutôt celle dont il convient de montrer la construction sociale, et la fonction de cette construction, est le « regroupement des individus selon le simple critère de l'identité patronymique », manière, pour une enquête lignagère, de ne pas présupposer l'objet de son étude. Or, justement, dans le corpus concernant les Thüngen, si le terme le plus fréquent dans le champ sémantique de la parenté est bien Geschlecht (lignage), c'est de fort près qu'il est suivi par Freundschaft. Ce n'est pas le moindre mérite de Joseph Morsel que de montrer combien ce dernier terme qui, en allemand actuel, signifie « amitié » (sens que lui donnent également, principalement, les ouvrages de lexicologie historique), relève essentiellement du champ de la parenté ; mais de façon complexe, puisque renvoyant avant tout à l'affinité (en englobant aussi la consanguinité), et

${ }^{3}$ - L'ouvrage est en effet riche des résultats de travaux menés en parallèle par Joseph Morsel avec une autre méthode, déterminant des populations d'étude plus vastes (l'ensemble des nobles appartenant à la même région que les Thüngen). Je renverrai au fur et à mesure à ces articles, dans la mesure où ils sont inclus dans le propos.

${ }^{4}$ - Un tel résultat n'a certes pu être obtenu que grâce à une réflexion novatrice et exigeante - mais l'on aurait tort de supposer que la base n'en est pas une érudition parfaite, tant pour le rassemblement des sources que pour la critique des documents. On en sait d'autant plus gré à l'auteur qu'il présente le matériau analysé dans de volumineuses annexes (170 pages de tableaux, cartes et éditions de sources) rassemblant une information considérable et rare. 
puisqu'il inclut aussi des relations non parentales. L'auteur analyse cette pluralité des sèmes comme l'indice de ce que, au Moyen Âge, «la parenté comme relation n'a qu'une faible spécificité par rapport aux autres rapports sociaux » (ce qu'il montre aussi à propos d'un terme comme Verwandt, qui, à l'inverse, désigne aujourd'hui exclusivement les relations de parenté), et conclut que «la Freundschaft est conçue comme le principe de base de tout rapport social positif (c'est-à-dire construisant des agrégats) ». L'analyse, nous semble-t-il, doit toutefois être poussée plus avant, en partant des éléments dégagés par J. Morsel : d'une part, Freundschaft renvoie à des relations de types variés (entre affins, entre consanguins, entre non-parents) ; d'autre part, l'affinité est son sème principal. Le premier point signifie que Freundschaft renvoie non à l'existence d'une relation formelle (par exemple le fait d'être affins), mais à une relation pratique : l'entraide. Comme celle-ci peut voir le jour dans des contextes variés, Freundschaft désigne les relations les plus diverses, mais n'est pas un terme générique pour chacun de ces types de relations : tout affin, par exemple, peut être mais n'est pas forcément un Freund. Néanmoins, son champ d'application est principalement parentélaire, et avant tout relatif à l'affinité ; ainsi, Freundschaft renvoie à la parenté pratique (et à elle seule dans le champ de la parenté), qui était manifestement comprise comme centrée sur l'affinité. Comme, par ailleurs, aucun autre terme ne désigne spécifiquement l'alliance comme rapport (ce qui n'est pas vrai de la consanguinité, au moins agnatique), qui n'est donc pas censée pouvoir être autre chose que Freundschaft, ce concept renvoie moins à cette manipulation de la parenté - à laquelle nous a habitués la sociologie contemporaine - qu'est la distinction entre parents pratiques et parents objectifs qu'à une manipulation d'une part, du mode d'exercice de la parenté (celle-ci étant implicitement désignée comme ne pouvant et ne devant être que pratique, la distinction parenté pratique/parenté objective ne doit pas avoir de raison d'être) ${ }^{5}$; d'autre part, des structures de la parenté (au centre desquelles est posée

5 - Freundschaft revient à dire qu'il n'est d'autre parenté acceptée que pratique : la parenté n'a pas de fonction, pas de possibilité d'être reconnue, pas de justification autre que son caractère pratique. Est alors paradoxal, pour le lecteur coutumier de l'allemand moderne, le fait suivant : tandis que Freundschaft, quoique non exclusivement relatif aux des relations de parenté, y trouve tout de même son champ d'application principal, Verwandtschaft renvoie pour sa part essentiellement à des relations autres que de parenté : relations de hasard, liées à l'appartenance à un même ensemble (par exemple une châtellenie), et connotées dépréciativement par l'emploi préférentiel comme terme de domination. Si les Verwandten, toutefois, peuvent parfois être des parents, c'est qu'il était nécessaire de présenter comme un repoussoir les relations de parenté non pratiques, purement formelles, non productrices d'un agir commun. En effet, ce n'était qu'en tant que la parenté, et tout 
l'alliance, mais d'une façon souple qui n'exclut en rien les autres relations de parenté) ; enfin, de la fonction de la parenté (puisque celle-ci est présentée comme le cœur de la Freundschaft, c'est-à-dire des relations d'entraide). En d'autres termes, la Freundschaft, d'une si grande importance empirique dans ce corpus textuel relatif à des nobles, signifie que l'alliance matrimoniale est la relation privilégiée au sein de ce groupe social, les autres types de relations s'organisant autour d'elle en compléments homothétiques (puisqu'ils sont subsumés sous la même notion). L'apparente confusion du terme recouvre donc une fonction sociale fort claire.

Ce rôle central imputé à l'alliance se comprend lorsque l'on considère non plus les termes qui désignent la parenté (comme relation), mais ceux par lesquels sont nommés des parents (concrets) : ils se limitent, pour ce qui est des parents au-delà du premier degré canon, à Vetter (consanguin) et Schwager (affin). Un tel regroupement, sous un seul et même terme, d'un aussi grand nombre de parents classificatoires distincts a en effet pour fonction de réaliser, par l'alliance, l'intégration dans un réseau parentélaire vaste et indifférencié, qui devient dans sa totalité et sans différences de degrés celui de l'allié, puisque les Vetter du conjoint deviennent tous des Schwäger ${ }^{6}$. L'alliance est donc mise en contact immédiate de deux ensembles parentaux incomparablement plus larges que ceux des familles nucléaires des deux époux, ce qui explique l'importance qui lui est accordée.

L'importance de l'alliance, toutefois, n'est pas assurée seulement par un discours qui subsume agnats et cognats sous une Freundschaft méliorative au centre de laquelle se place l'affinité, mais elle l'est aussi par le mode de réalisation concret des alliances ${ }^{7}$. Leur première caractéristique est l'écart entre nubilité et nuptialité (les femmes se marient vers 20 ans, les hommes vers 30 ans) qui, couplé à la basse espérance de vie ( 35 ans pour les femmes, 45 pour les hommes), a pour conséquence que, au moment où ils atteignent leur majorité (14 ans), les

particulièrement l'alliance, était génératrice d'une parenté non pas seulement objective mais pratique - et donc d'un groupe - qu'elle pouvait assumer son rôle fondamental dans le fonctionnement du groupe nobiliaire tel qu'il se redéfinit au $\mathrm{xv}^{\mathrm{e}}$ siècle.

${ }^{6}$ - Ce vocabulaire extrêmement sommaire est d'autant plus significatif qu'il n'excluait nullement une perception fine des différents degrés de parenté ; simplement, ceux-ci n'étaient pas lexicalisés, et leur connaissance n'était mobilisée que lors de situations spécifiques et rares (l'héritage, le mariage en raison des interdits canoniques), mais pas dans le quotidien des désignations.

${ }^{7}$ - Le problème me paraît central quant au fonctionnement de l'alliance. Joseph Morsel le traite, mais à propos d'un autre aspect (le veuvage et la transmission des biens). 
fils ${ }^{8}$ sont déjà orphelins, ou vont bientôt l'être ; le système de parenté peut donc être défini comme un processus de déstructuration volontaire des relations du premier degré (du comput romain $)^{9}$, sans importance pratique puisqu'elles ne peuvent être relations entre adultes (c'està-dire d'action). On retrouve ici ce que l'on avait vu à propos de Schwäger et Vetter, mais l'on est désormais capable d'en rendre compte : si l'alliance est entre parentèles et non entre familles nucléaires, c'est que celles-ci ont déjà disparu au moment du mariage des enfants. Tout aussi fondamentale est la seconde caractéristique concrète des alliances, puisqu'elle détermine la parenté pratique des orphelins; en effet, si l'on considère non plus l'écart général entre nubilité et nuptialité mais le caractère différentiel de cet écart entre les hommes et les femmes, c'est-à-dire l'âge au mariage plus élevé des hommes, on voit que, au moment de leur majorité, si les fils perdent leurs parents ainsi que leurs oncles paternels, s'offrent en revanche à leur appui leurs oncles maternels ${ }^{10}$ (qui ont encore une dizaine d'années à vivre), puis, remplaçant ces derniers après leur mort, les maris et beaux-frères de leurs sœurs (de dix ans plus âgés qu'eux-mêmes), et finalement leurs propres beaux-frères : successivement, donc, l'alliance, sous des modalités différentes, est seule en mesure de procurer au jeune noble l'appui de parents plus âgés. Le bon fonctionnement des relations d'alliance (c'est-àdire le fait qu'elles soient Freundschaft) est donc essentiel à la reproduction d'un groupe qui, pourtant, insiste discursivement sur la parenté agnatique (Geschlecht étant le premier terme, en nombre d'occurrences, dans le champ sémantique de la parenté).

Dans ces conditions, l'on voit que ce qui posait problème était l'existence d'une identité propre au groupe des agnats, puisque, au niveau des interactions directes entre agents, ce groupe n'avait qu'une réalité restreinte - raison pour laquelle c'est justement sur la dimension

${ }^{8}$ - On ne s'intéresse ici qu'aux fils parce qu'ils sont les seuls à jamais devenir majeurs, les filles ne faisant que passer de la tutelle de leur père à celle de leur époux.

9 - Ce dont témoigne l'absence de termes désignant spécifiquement les groupes reliés par ces relations (aucun mot ne correspond à la famille nucléaire), ce qui contraste avec l'existence de termes pour désigner les groupes de parents du deuxième degré (Geschwister).

10 - C'est, à notre sens, sans doute parce que le recours aux oncles paternels a de ce fait une fonction pratique moindre, que le terme qui les désignait à l'origine exclusivement (vetter) a pu prendre un sens beaucoup plus générique (en l'occurrence: consanguin), parce que le mot était comme libre, ce qui n'était au contraire pas le cas du vocable désignant les oncles maternels (oheim). Ainsi dans le corpus de Joseph Morsel, si les oncles paternels ne représentent que $2 \%$ des occurrences de vetter pour lesquelles peut être reconstruit le degré de parenté, par contre les oncles maternels fournissent $60 \%$ des oheime. 
agnatique qu'a porté l'effort discursif, empruntant non seulement l'approche lexicale, mais aussi l'anthroponymie (patronymie unifiée des agnats ${ }^{11}$ ) et les media non linguistiques (héraldique et emblématique). Au niveau des prénoms masculins, les logiques sont plus complexes, d'une part, parce que coexistent les stratégies d'intégration à la noblesse (par reprise des prénoms chevaleresques répandus dans tous les lignages et qui singularisent les nobles par rapport aux autres groupes sociaux) et d'identification au lignage; d'autre part, parce que l'anthroponymie lignagnère procède de deux manières bien distinctes (par la collation de prénoms spécifiques au lignage; et par la transmission des prénoms de l'aïeul au petit-fils) ; enfin, parce que ces différents éléments peuvent se combiner, la transmission d'un prénom chevaleresque de deux générations en deux générations renvoyant à la continuité agnatique aussi bien qu'elle assure l'intégration à l'ensemble de la noblesse. On voit donc que les différents niveaux de l'anthroponymie (rendus possibles par l'apparition, largement antérieure à la période étudiée, de la dénomination double) permettent la prise en charge de discours multiples par un même marqueur d'identité (l'anthroponymie), mais que, par ailleurs, un élément (le prénom) autorise également à lui seul la prise en charge de plusieurs discours identitaires ${ }^{12}$. L'insistance agnatique se repère de façon particulièrement nette au niveau de l'héraldique, caractérisée (à l'inverse d'autres zones de la chrétienté médiévale) par l'absence totale des brisures (singularisant les lignées au sein du lignage, insistance patrilinéaire donc) et la rareté de l'écartèlement (singularisant les individus en fonction de l'alliance dont ils procèdent) : non que cette dernière singularisation ne puisse s'opérer, mais elle préserve l'intégrité du blason, ainsi dans l'art funéraire ${ }^{13}$. L'identité ainsi obtenue entre

11 - Sur le patronyme, voir Joseph Morsel, «Changements anthroponymiques et sociogenèse de la noblesse en Franconie à la fin du Moyen Âge », in M. Bourin et P. Chareille (dir.), Genèse médiévale de l'anthroponymie moderne, III, Enquêtes généalogiques et données prosopographiques, Tours, Publications de l'Université de Tours, 1995, pp. 89-119.

12 - Sur les prénoms nobiliaires, voir Joseph Morsel, « De l'usage politique et social des prénoms en Franconie à la fin du Moyen Âge », in P. Contamine, T. Dutour et B. SchnerB (dir.), Commerce, finances et société (XI-XVI siècles). Recueil de travaux d'histoire médiévale offert à M. le Professeur Henri Dubois, Paris, Presses de 1'Université ParisSorbonne, «Cultures et civilisations médiévales-9 », 1993, pp. 379-393

13 - L'absence de transmission aux enfants des armoiries écartelées, de même que la présence sur les tombeaux des armes non des deux géniteurs mais des quatre aïeuls, corroborent ainsi le caractère secondaire du couple - particularité remarquable vue la place donnée à l'alliance, en aucune façon niée, puisque ce sont les armes des quatre aïeuls qui 
les différents systèmes de signes, tous mis au service de l'expression d'une identité lignagère, était renforcée par l'utilisation conjointe des différents systèmes dans un même medium : il en est ainsi du sceau, puisque le sceau armorié accompagné d'une légende anthroponymique, et souvent timbré, est le type le plus fréquent. On retrouve d'ailleurs l'expression consciente de cette identité des media dans des expressions telles que « name, stamm, schild und helm ».

Ces pratiques discursives reliant des lignées aux degrés de parenté de plus en plus lointains au fur et à mesure que passe le temps sont redoublées d'autres pratiques, non discursives. Rendues possibles par ces discours (par les représentations qu'ils engendrent, qui forment le schème d'actions), elles contribuent à reproduire leurs conditions de possibilité. Ainsi, l'institution d'indivisions lignagères portant sur des châteaux, dont les parts ne peuvent se transmettre qu'aux mâles (ce qui, au moment de la constitution des premières indivisions, dans le second tiers du XIv ${ }^{\mathrm{e}}$ siècle, allait à l'encontre de la coutume successorale) et ne peuvent être vendues en dehors du Geschlecht ${ }^{14}$ (disposition là aussi dérogatoire au Landrecht); ou les mariages entre Thüngen éloignés de plus de quatre degrés canoniques, c'est-à-dire ceux qui peuvent se marier entre eux sans aller à l'encontre des proscriptions ecclésiastiques, et ceux dont les liens de parenté deviennent si distendus qu'il est nécessaire d'ainsi les raffermir (fonctionnement qui rappelle celui dégagé par Gérard Delille pour d'autres lieux et d'autres temps ${ }^{15}$; et enfin le choix d'un lieu d'inhumation commun à tous les membres du lignage ${ }^{16}$.

Cet ensemble de pratiques visant à homogénéiser un ensemble d'agnats aux degrés de parenté souvent lâches permet de « faire apparaître comme un groupe de parenté ce qui est un

figurent sur les dalles funéraires.

14 - J'emploierai désormais systématiquement Geschlecht pour éviter le terme de « lignage », manière de rappeler que la parenté, dans le groupe nobiliaire, n'est structurée agnatiquement que, pour l'essentiel, au niveau des pratiques discursives. La parenté comme interaction est, elle, cognatique, et a pour structure fondamentale non la descendance mais l'alliance, non le lignage mais la parentèle.

15 - Gérard Delille, Famille et propriété dans le royaume de Naples (XV-XIX siècle), Roma, École Française de Rome, 1985.

16 - Si le lieu d'inhumation commun contribue à l'entretien de la mémoire du lignage, en revanche les pratiques "mémoriales" explicites sont elles vouées à la mémoire de l'alliance, le conjoint étant toujours compris parmi les bénéficiaires des messes anniversaires, de même que, fréquemment, les géniteurs du fondateur comme de son conjoint. Les pratiques funéraires articulent donc les deux dimensions de la parenté nobiliaire. 
groupe de succession (ou un groupe successoral), c'est-à-dire de faire disparaître le problème de la reproduction du pouvoir seigneurial derrière la fiction d'une continuité biologique ${ }^{17} »$, et, par là, de supprimer la contradiction entre la structuration de la parenté autour des liens d'alliance et la non-pertinence (qu'il reste à expliquer) de ces liens par rapport aux problèmes successoraux. Si le ciment de la Freundschaft se trouve dans une structure de parenté qui, à travers le jeu sur les âges des conjoints, rend inévitable la co-action avec les affins, celui du Geschlecht réside dans des discours dont l'efficace n'est pas moindre et la portée pratique tout aussi grande (puisque déterminante pour les questions successorales) ; l'essentiel étant de voir que ces deux dimensions de la parenté, loin d'être concurrentes, se renforcent l'une l'autre dans la mesure où c'est la solidité même du Geschlecht, produite discursivement, qui assure l'efficacité de la Freundschaft puisque seule cette solidité permet, par le mariage, de s'allier non pas à une famille nucléaire, mais à l'ensemble de la parentèle du conjoint, et donc à un groupe suffisamment important et soudé pour que son appui ait du poids.

De cette complémentarité témoigne exemplairement l'évolution de l'anthroponymie des épouses : alors que, jusque vers 1400, l'anthroponymie féminine (et aussi bien les pratiques sigillantes des épouses - ou plutôt leur large absence, due à l'utilisation du sceau de l'époux) ne représentait pas le mariage comme une alliance, mais comme l'adjonction à un Geschlecht d'un nouveau membre féminin qui s'y intégrait pleinement (à tel point que l'on est généralement incapable de déterminer aujourd'hui le Geschlecht d'origine des épouses), au

17 - J. Morsel, La noblesse contre le prince..., op. cit., p. 00. L’idée, fondamentale (et reprise d'Anita Guerreau-JaLABert, «El sistema de parentesco medieval : sus formas (real/espiritual) y su dependencia con respecto a la organización del espacio », in R. PAsToR (dir.), Relaciones de poder, de producción y parentesco en la edad media y moderna: aproximación a su estudio, Madrid, CSIC, 1990, pp. 85-105), est que la parenté, dans sa dimension agnatique, était subordonnée à une logique seigneuriale qu'elle n'avait pour objet que d'assurer. Si les relations agnatiques assurent la transmission du pouvoir seigneurial, ce n'est pas que cette transmission obéisse aux structures de la parenté, mais que ces dernières ont été (pour ce qui est de leur dimension discursive) structurées en fonction des contraintes de cette transmission; la logique de la parenté est en fait la topolinéarité, soit une logique spatiale et non parentale. La clé du discours agnatique se trouve donc non dans les structures internes du groupe nobiliaire mais dans la relation dominantsdominés, et l'on voit alors que la contradiction entre discours agnatique et structures cognatiques effectives, loin d'être une faiblesse, permet, grâce aux relations de parenté, d'assurer et la cohésion du groupe dominant (par l'alliance) et la reproduction de sa domination (par l'illusion créée d'une continuité seigneuriale). 
$\mathrm{Xv}^{\mathrm{e}}$ siècle l'anthroponymie des épouses prend la forme « $\mathrm{X}$ von $\mathrm{Y}$, née $\mathrm{Z}$ ». Cette transformation exprime le passage d'une présentation du mariage comme assurant la reproduction du Geschlecht épouseur, à une conception du mariage en tant qu'alliance entre deux Geschlechter - ce lien ne pouvant être durablement manifesté que si l'épouse conserve, juxtaposée à celle acquise par son mariage, son identité lignagère originelle. L'appartenance conjointe à deux Geschlechter est, toutefois, impossible, et la juxtaposition de deux identités lignagères dans l'anthroponymie des épouses n'est que le signe de leur non-intégration à l'un et à l'autre lignages, concrètement manifestée par les dispositions juridiques qui excluent l'épouse tant de son Geschlecht d'origine (exhérédation des filles dotées - or la fonction même du Geschlecht est la transmission des biens) que de son Geschlecht d'accueil (en cas de remariage consécutif à un veuvage, la femme perd ses enfants qui restent, de même que sa contre-dot, dans le Geschlecht de son mari). La fonction des épouses consiste donc simultanément à signifier l'alliance entre Geschlechter, à manifester le caractère unique de l'identité lignagère et à renvoyer à une identité non lignagère : l'identité de l'épouse, exclue de ses deux Geschlechter d'origine et d'accueil, peut en effet se définir comme une identité noble « pure », non entachée de l'appartenance singularisante à un Geschlecht. Or, justement, la fonction de l'alliance, réalisée par les femmes et par elles seules (puisque ce sont elles, et non les hommes, qui passent d'un Geschlecht à l'autre, l'uxori-localité étant inconnue), est de faire exister la noblesse en tant que réseau, en tant que groupe supra-lignager ; les épouses se devaient donc de n'être que nobles, et d'assumer l'impossibilité de leur appartenance lignagère.

Ce problème de l'alliance comme mode d'existence de la noblesse en tant que groupe aurait dû amener Joseph Morsel à s'intéresser plus qu'il ne l'a fait (mais on lui sait gré d'avoir dégagé la signification du problème) à d'autres pratiques, ritualisées, qui symbolisent ce fonctionnement. L'une, déjà bien diffusée à la fin du Moyen Âge (notamment en lien avec cette autre qui définit la noblesse, le tournoi) et appelée à un succès plus considérable encore, est le bal. Il n'est en effet que la mise en scène de la possibilité de l'alliance au sein d'un groupe, sans exclusive (l'exclusive étant reportée au niveau de la définition du groupe autorisé à participer à cette festivité $)^{18}$. N'aurait-il pas fallu s'interroger sur la transformation des usages sociaux de la danse, afin de voir dans quelle mesure elle était homologue, dans son contenu comme dans sa chronologie, aux transformations repérées dans le champ identitaire

\footnotetext{
18 - Pratique que l'on retrouve chez les patriciens nurembergeois, avec le Tanzstatut de 1521 qui établit la liste des lignages seuls autorisés à participer aux bals organisés à l'Hôtel de ville.
} 
(anthroponymie et sigillographie)? Il fait néanmoins peu de doute que l'émergence du bal renvoie au positionnement non lignager des épouses, dans la mesure où, n'étant plus liées exclusivement à un Geschlecht (celui de leur époux), il leur devient possible de représenter symboliquement la circulation des femmes entre les Geschlechter (c'est-à-dire entre les hommes) par le biais de cette « forme d'appariement changeant » qu'est la danse ${ }^{19}$.

Revenons au problème de l'alliance matrimoniale comme structuration de l'unité du groupe noble, mais pour l'aborder sous un autre angle. J. Morsel insiste sur ce point à de nombreuses reprises : au Moyen Âge les relations sociales s'expriment et se réalisent par la circulation des biens. De ce fait, si le discours qu'est le Geschlecht a pour but la transmission agnatique des seigneuries, la relation sociale qu'est la noblesse (en tant que regroupement de Geschlechter) est, elle, manifestée par la circulation des biens dotaux. Celle-ci est organisée par trois contraintes : les héritiers des dots existantes sont non le mari mais les enfants (y compris si la femme meurt avant son époux); les dots nouvelles sont constituées pour ses filles par l'époux sur ses biens propres ; la troisième contrainte étant celle, déjà évoquée, de la différenciation sexuée de l'âge au mariage. Comme les filles sont mariées plus précocement que les garçons, et comme, de ce fait, la mère est toujours plus jeune que le père, les biens dotaux ne reviennent qu'aux fils, puisqu'à la mort de leur mère les filles sont déjà mariées, c'est-à-dire dotées et donc exhérédées ; au contraire, l'âge au mariage élevé des garçons compense la jeunesse de leur mère, ce qui signifie que, au moment du mariage de leurs propres filles, ils sont en mesure de les doter grâce aux biens reçus de leur mère, déjà décédée. La conjonction des trois contraintes permet donc de faire circuler les biens sur un modèle radicalement différent de celui de la transmission agnatique, puisque, au lieu de circuler d'homme à homme du même Geschlecht, les biens dotaux passent non de femme en femme mais d'épouse en épouse, c'est-à-dire de Geschlecht en Geschlecht (puisque l'uxori-localité

19 - J. Morsel, La noblesse contre le prince..., op. cit., p. 00. Auparavant au contraire, la littérature courtoise figurait l'impossibilité de la circulation des femmes au sein d'une aristocratie profondément scindée en sous-groupes hiérarchisés. On voit d'ailleurs par là que les hypothèses de Joseph Morsel sur la fonction de la circulation des femmes dans la genèse du groupe nobiliaire permettraient d'étudier sous un angle d'histoire sociale l'évolution de l'adultère noble, pratique sociale qui, semble-t-il, se développe au $\mathrm{XVI}^{\mathrm{e}}$ siècle, et, surtout, prend des formes institutionnalisées, particulièrement autour des princes, qui trouvent dans le concubinage officialisé le moyen d'exprimer leur appartenance à la noblesse. On devrait donc pouvoir observer une importance différentielle de la place des maîtresses princières dans les régions où la genèse de l'État s'est effectuée sans trop d'encombres et dans celles où elle a échoué. 
est inconnue). Les porteurs et les directions de la circulation diffèrent donc. Cela est d'autant plus significatif qu'il n'y avait là rien qui fût structurellement inévitable, puisqu'il y avait bien un mélange temporaire des biens provenant du père et de la mère, qui rendait possible la constitution des dots avec des biens hérités du père ; en effet, les deux lignes de circulation se croisent au niveau des hommes, par ailleurs seuls responsables de l'affectation des dots. Il convient, pour rendre visible l'ensemble des implications de cette organisation des circulations dotales, de préciser que la constitution du patrimoine des épouses exhérédées n'est pas seulement le fait de leur père, par le biais de la dot, mais tout autant celui de leur beau-père, par une contre-dot strictement équivalente ; la conséquence en est qu'un époux, lorsqu'il constitue la dot de ses filles, puisqu'il y emploie les biens qui lui viennent de sa mère, y affecte donc des biens provenant du Geschlecht de sa mère mais aussi du Geschlecht de son arrière-grand-mère paternelle (puisque c'est avec les biens venant de sa propre mère que son grand-père paternel a constitué la contre-dot de sa bru ${ }^{20}$. Ainsi les biens qui circulent à l'occasion d'un mariage ne manifestent-ils pas seulement l'alliance entre deux Geschlechter, mais réactualisent les alliances antérieures avec d'autres Geschlechter (et l'on comprend alors pourquoi, sur les monuments funéraires, les armes représentées sont celles non pas des deux parents du défunt, mais de ses quatre aïeuls) ; une telle alliance, telle qu'elle se manifeste à travers la circulation des biens, n'est jamais affaire de Geschlechter autonomes, mais de Geschlechter déjà pris dans un réseau d'alliances, de parentèles donc, de réseaux structurés non par la descendance mais par l'alliance : celle-ci ne peut donc que manifester l'appartenance à la noblesse. L'on a ici la raison pour laquelle les négociations matrimoniales sont effectuées non par des représentants des deux Geschlechter concernés, mais par des membres des deux parentèles ${ }^{21}$. Par ailleurs, cette question de la confusion de biens provenant de plusieurs Geschlechter dans le patrimoine des épouses nous permet de rendre compte d'un aspect jusqu'ici non expliqué de l'organisation de l'alliance: non pas la différenciation

${ }^{20}$ - Cette dernière dimension a échappé à la sagacité de Joseph Morsel - mais l'essentiel était de dégager le circuit spécifique de la circulation des biens dotaux.

${ }^{21}$ - Dans tous les cas, l'alliance n'est pas négociée par le père, de toute façon souvent déjà décédé au moment du mariage de ses enfants (ses fils plus encore que ses filles) : l'alliance ne doit pas pouvoir être réalisée par celui qui représente le principe patrilinéaire (soit le principe anthropologique inverse de celui qui fait de l'alliance une valeur et non une simple nécessité reproductive). En effet, les deux modes de fonctionnement de la parenté nobiliaire sont l'alliance et le principe agnatique (le lignage), en aucun cas la patrilinéarité (la lignée). 
sexuée de l'âge au mariage, mais son ampleur ; en effet, celle-ci correspond à peu près à la différenciation sexuée de l'espérance de vie (plus faible chez les femmes), ce qui signifie que l'écart entre l'âge des époux est nettement plus grand que l'écart entre leurs dates de décès; par conséquent, les veuvages féminins longs sont rares, ce qui permet de limiter les remariages, qui provoqueraient le retour dans les deux Geschlechter respectifs de la dot et de la contre-dot, et empêcheraient donc la fusion, dans les dots des générations ultérieures, des biens de plusieurs Geschlechter - c'est-à-dire la manifestation de la dimension supralignagère, nobiliaire par excellence, de l'alliance.

Que l'on considère l'obligation faite aux agents mâles de se reposer davantage sur leurs affins que sur leurs consanguins ou la circulation des biens dotaux, la différenciation sexuelle de l'âge au mariage nous apparaît revêtir un rôle central, puisqu'elle est la condition de possibilité de ces logiques. Il serait bien évidemment absurde d'y voir la cause de ces modes de fonctionnement, puisque cette différenciation n'était que le moyen (extraordinairement efficace, puisque, d'une part, il opérait comme contrainte, et que, d'autre part, il assumait simultanément des fonctions différentes) employé par les nobles afin d'aboutir à un système qui, pour faire circuler les biens principaux selon un principe agnatique (qui n'est lui-même que le mode de réalisation de la topo-linéarité), n'en conservait pas moins à l'alliance un rôle fondamental. Il reste à comprendre pourquoi ce double mode de fonctionnement de la parenté a pu paraître nécessaire aux nobles franconiens : parce que, et telle est l'hypothèse de Joseph Morsel, tout se passe comme si seule la conjonction de ces deux principes distincts avait permis de placer les princes dans un piège tel qu'ils ne pussent mener à bien la construction d'un État qui aurait transformé les nobles en sujets, et empêcher les nobles de se constituer en un groupe unifié et structuré, ainsi capable de s'opposer victorieusement aux princes. Il convient donc de se tourner désormais vers les relations entre les nobles (tendant à former une noblesse) et leurs seigneurs (tendant à se transformer en princes).

\section{Les nobles et leurs seigneurs}

À l'encontre d'une historiographie qui ne veut souvent voir dans les liens féodaux de la fin du Moyen Âge qu'un ensemble de formes creuses et/ou dévoyées de leur fonction (bastard feudalism), J. Morsel montre que le lien féodal ne devient fondamental qu'au $\mathrm{Xv}^{\mathrm{e}}$ siècle ; alors en effet intervient une augmentation quantitative et qualitative extraordinaire des fiefs détenus par les Thüngen : de 1300 à 1525, leur nombre quintuple, et les châteaux (à une exception près) n'apparaissent parmi ces fiefs qu'à partir du second quart du $\mathrm{xv}^{\mathrm{e}}$ siècle. La raison n'en est 
pas tant que les Thüngen, par rachats ou héritages, remplaceraient d'autres vassaux (transformation qui ne concernerait que les Thüngen, et non les cours féodales auxquelles ils appartiennent), mais qu'ils reprennent massivement en fief leurs alleux (essentiellement au cours des années 1425-1450, puis 1500-1525). Ces reprises sont pour eux le moyen de s'intégrer au plus haut niveau à l'une des cours féodales les plus importantes de Franconie, celle des margraves de Brandebourg-Ansbach, cour dont ils étaient jusque-là totalement absents pour des raisons géographiques; en effet, cette cour était implantée en Moyenne et Haute-Franconie, et non dans la région de Basse-Franconie où étaient concentrées les possessions des Thüngen. Leurs nombreuses reprises en fief ne sont donc pas à interpréter comme une perte d'indépendance des Thüngen mais comme une réorganisation de leurs liens avec les seigneurs vassaliques, effectuée au moment même (le $\mathrm{xv}^{\mathrm{e}}$ siècle) où ceux-ci cherchent à se transformer en princes territoriaux. Les Thüngen, par le choix excentré de la cour féodale où ils effectuent leurs reprises en fief, manifestent ainsi leur refus de la territorialisation. Le fief passe donc, du début $d u \mathrm{XIV}^{\mathrm{e}}$ siècle au début du XVI ${ }^{\mathrm{e}}$ siècle, d'une concession faite par un grand local à des nobles non titrés dans leur zone d'implantation, à une reprise par les nobles de leurs biens allodiaux auprès d'un prince excentré : le sens de la circulation et ses acteurs s'inversent ${ }^{22}$.

L'interprétation semblerait donc devoir être la suivante, particulièrement si l'on prend en compte le fait que le second quart $\mathrm{du} \mathrm{Xv}^{\mathrm{e}}$ siècle est le moment du grand conflit armé des Thüngen contre le principal seigneur féodal local, l'évêque de Wurtzbourg : les Thüngen jouent des conflits entre princes franconiens pour préserver leur autonomie - mais le coût en est une diminution de la part allodiale de leurs possessions (qui devient quasiment nulle en ce qui concerne les plus importantes, les châteaux). Une telle interprétation, toutefois, revient à supposer que les relations féodales auraient été les seules par lesquelles les Thüngen pouvaient se rapprocher d'un prince et s'assurer de son soutien. Or, si le $\mathrm{xv}^{\mathrm{e}}$ siècle paraît bien, en Franconie, représenter l'âge d'or de la féodalité, il est tout autant celui au cours duquel se développent les relations contractuelles de service (armé et administratif) - et, à considérer les

22 - Cette évolution n'est nullement spécifique aux relations des Thüngen avec les margraves, comme le montre l'étude d'ensemble d'une autre cour féodale de l'espace des Thüngen: le premier registre des fiefs de l'évêque de Wurtzbourg (1303-1313) ne documente qu'une reprise en fief, tandis que celui de 1507-1519 en connait quarantequatre (Hans-Peter Baum, Der Lehnhof des Hochstifts Würzburg im Spätmittelalter (13031519): Eine rechts- und sozialgeschichtliche Studie, Universität Würzburg, Habilitationschrift, 1990). 
relations entre les Thüngen et les margraves, l'on s'aperçoit d'ailleurs que celles-ci précèdent chronologiquement les reprises en fief. La fonction de la reprise en fief n'était donc pas seulement le rapprochement avec un prince excentré, puisque d'autres formes de relation, moins contraignantes pour les nobles, auraient aussi bien pu être utilisées pour ce faire (et l'ont effectivement été), et que des reprises, quoique moins nombreuses, ont également eu lieu au profit des seigneurs féodaux locaux.

La transformation de la relation des nobles aux fiefs renvoie en effet tout autant à la modification du droit des fiefs opérée au $\mathrm{xv}^{\mathrm{e}}$ siècle. Antérieurement, le droit des fiefs s'opposait au droit coutumier (Landrecht, dont dépendent les alleux) dans la mesure où les fiefs n'étaient transmissibles qu'aux héritiers mâles directs (Mannlehen, ce qui impliquait le retour au seigneur féodal en l'absence de tels héritiers), tandis que les alleux devaient être partagés entre tous les héritiers directs, de quelque sexe qu'ils soient. $\mathrm{Au} \mathrm{Xv} \mathrm{v}^{\mathrm{e}}$ siècle, si le droit coutumier ne change pas, on se met en revanche à distinguer entre fiefs roturiers (tenus soit sous la forme du Mannlehen classique, soit sous la forme du Zinslehen transmissible aux cognats) et fiefs chevaleresques (transmissibles aux agnats). Le fief devient ainsi le moyen d'assurer le succès de la topo-linéarité en tant qu'elle passait par la construction lignagère (agnatique), qui avait donc besoin des princes pour s'opérer; inversement, cette question des fiefs permet de comprendre que l'objet de la construction lignagère est bien d'assurer un mode particulier de transmission des biens - et, en dernier ressort, renvoie au problème de la reproduction sociale. En effet, deux stratégies de reproduction (s'excluant réciproquement) étaient possibles : l'une, fondée sur la multiplication des membres du groupe afin d'éviter son extinction biologique, et donc le passage de ses biens à un autre groupe de parenté, n'était possible que si les biens pouvaient circuler entre les sous-groupes créés par cette multiplication (les lignées), ce qui rendait nécessaire l'établissement de la succession agnatique ; l'autre était au contraire basée sur la limitation du nombre des membres du groupe afin d'éviter l'éclatement des biens. Ces deux stratégies ont été utilisées différentiellement au sein de l'aristocratie: tandis que les nobles non titrés, issus de la ministérialité, s'engouffraient dans la première, reprenant massivement leurs biens en fief, une telle stratégie était impossible pour les nobles titrés d'origine « libre » (Edelfreien, nobiles), dont la position spécifique, qui tenait à leur absence de dépendance à l'égard d'autres puissants (et à la constitution d'une cour féodale), devait impérativement être conservée pour pouvoir former le socle de prétentions à la construction d'un État territorial : la revendication d'une qualité princière radicalement différenciée de celle des sujets ne pouvait être tenue que si l'on était soi-même libre de liens de subordination. On voit alors que le «hasard biologique»si 
souvent mis en avant pour expliquer pourquoi telle lignée titrée et non telle autre est finalement parvenue au statut princier (les échecs étant le plus souvent simplement dus à l'extinction), s'il renvoie bien à une réalité, renvoie effectivement à une réalité biologique, mais socialement construite ${ }^{23}$.

La féodalisation massive des nobles non titrés en Franconie à la fin du Moyen Âge répond donc à deux logiques distinctes : s'allier à un prince excentré pour contrer les tentatives de sujétion au prince local, et assurer la transmission des possessions à l'intérieur du seul lignage. Cette double logique implique des protagonistes différents, comme le montre exemplairement le cas des Thüngen : tandis que, au moment de leur affrontement avec le prince local (l'évêque de Wurtzbourg), c'est des lointains margraves de Brandebourg qu'ils reprennent leurs alleux en fiefs (dans le deuxième quart $\mathrm{du} \mathrm{xv}^{\mathrm{e}}$ siècle), une fois leur position ainsi assurée face à l'évêché, la reprise pour raison successorale peut parfaitement s'opérer auprès du prince local (premier quart du $\mathrm{XVI}^{\mathrm{e}}$ siècle).

Le problème est alors de déterminer pourquoi les princes ont accepté de procéder à cette réorganisation de la succession aux fiefs alors même qu'elle leur était particulièrement défavorable, puisqu'elle limitait aux cas de commise les possibilités de retour du fief au seigneur. Mais la double logique même de ces reprises en fief permet de voir que la contradiction n'est qu'apparente, puisque c'est précisément dans la mesure où une partie de ces reprises en fief s'inscrivait dans le cadre des conflits entre princes (les évêques de Wurtzbourg et les margraves de Brandebourg se disputant tout au long du $\mathrm{Xv}^{\mathrm{e}}$ siècle la suprématie sur la Franconie) que ces reprises servaient aussi les intérêts des princes : en s'assurant ainsi des fidélités et des prérogatives dans les zones où ils étaient le moins implantés, non seulement les margraves renforçaient leur emprise sur l'ensemble de la Franconie, base préalable à la construction d'un État territorial, mais ils affaiblissaient la cohérence géographique du contrôle de leur concurrent princier. Ce n'est qu'à partir du moment où les deux protagonistes prirent conscience de leur échec réciproque (lié au fait qu'ils avaient tous deux usé des mêmes moyens féodaux) qu'ils acceptèrent de se partager la Franconie, vers 1495, ce qui certes obligea les nobles non titrés à recourir à d'autres moyens que la reprise en fief pour continuer à assurer leur autonomie ${ }^{24}$, mais n'entama en rien les

${ }^{23}$ - Et d'autant plus socialement construite que l'extinction n'est en fait jamais que l'absence d'héritiers mâles : les biens des lignées titrées qui tombent en quenouille passent, par les femmes, à d'autres lignées titrées, ainsi renforcées dans leurs efforts de construction d'une principauté. 
résultats qu'ils avaient déjà obtenus, soit une plurivassalité massive (par surcroît sans ligesse, inconnue dans la région).

Surtout, point que ne mentionne pas J. Morsel mais qui est la conséquence nécessaire de son propos (et qui serait resté parfaitement invisible sans son explicitation de la fonction de la reprise en fief), si les nobles, à partir du début $\mathrm{du} \mathrm{XvI}^{\mathrm{e}}$ siècle, peuvent effectivement trouver contre les princes de nouveaux moyens d'action, c'est, dans la mesure où ceux-ci reposent sur la constitution de la noblesse en groupe institutionnalisé (naissance de la Reichsritterschaft comme organisation politique), parce que la féodalisation de leurs possessions a posé les bases matérielles de cette unité. En effet, parce que le fief nouvelle manière faisait prévaloir la succession agnatique, il supprimait les motifs de conflits entre lignages au moment des héritage ${ }^{25}$; ces conflits étaient au contraire fort nombreux au XIV $^{\mathrm{e}}$ siècle, comme le montre notamment l'affrontement durable entre les Thüngen et les Hutten autour de l'héritage des Steckelberg - des membres des deux premiers lignages ayant épousé les héritières du troisième, dépourvu de descendant mâle direct; le fief, donc, n'assure pas seulement la

${ }^{24}$ - Et c'est parce que la reprise en fief perdait ainsi sa fonction «politique » (tout en gardant son rôle successoral) qu'elle pouvait désormais se faire auprès du prince local.

25 - On touche là un problème absolument essentiel, puisque cela signifie que la construction de l'unité du lignage ne se fait plus par l'opposition à d'autres lignages. Toutefois, le report des conflits successoraux du cercle des cognats (relevant de lignages différents) au cercle des agnats (relevant de lignées différentes du même lignage), s'il renforce la noblesse, par contre affaiblit le lignage. Il convient cependant de ne pas perdre de vue que, comme c'est l'appartenance des parties au lignage qui rend leur conflit possible parce qu'elle leur donne des droits sur la succession, celui-ci ne peut (sous peine de mettre en danger ces droits mêmes pour lesquels on est en conflit) remettre en cause le lignage. La succession agnatique permise par les fiefs non seulement donc supprime les conflits entre lignages (et permet ainsi le fonctionnement de la noblesse en tant que groupe uni), mais atténue aussi les querelles successorales par leur report au sein d'un autre type de groupe. Par ce passage du conflit du groupe des cognats au groupe des agnats, il prend des formes qui ne sont plus celles de la faide mais de l'arbitrage. On voit donc que l'on est passé d'un fonctionnement où l'identité lignagère, fragile parce que non appuyée sur les règles successorales, se renforçait par son opposition à d'autres lignages (à propos de la succession de lignages tiers), à un fonctionnement où l'identité lignagère, renforcée par les règles successorales, n'a plus besoin de s'opposer à d'autres lignages. Ainsi, c'est uniquement le lien entre lignage et fief nouvelle manière qui permet de ne plus faire jouer le lignage contre la noblesse. 
reproduction du lignage en tant que groupe successoral, mais aussi la reproduction de la noblesse en tant que juxtaposition pacifique de lignages : le fief, dans les formes spécifiques qu'il prend au $\mathrm{xv}^{\mathrm{e}}$ siècle, contient aussi bien le lignage que la noblesse (ou du moins leurs conditions de possibilité), et l'on voit que, paradoxalement, les deux formes qui assurent l'autonomie des nobles non titrés ont été construites grâce au prince. Le piège dans lequel ont été pris les princes, et qui les a empêchés d'intégrer les nobles à leur État naissant, est que, pour empêcher la constitution de ces nobles en une noblesse structurée et unie, capable de s'opposer à eux avec succès, il était nécessaire de la morceler en ses lignages constitutifs, et donc de renforcer l'emprise des structures lignagères. Le principal moyen à la disposition des princes pour ce faire était la féodalité, moyen qu'ils ne se sont pas privés d'employer, mais qui allait en fait à l'encontre de leurs intérêts dans la mesure où, pour renforcer les lignages, il confortait aussi bien leur intégration en une noblesse - qui se trouvait donc doublement raffermie, et dans son unité, et dans la puissance des sous-groupes qui la constituaient.

Plus largement, c'est parce que les petits nobles ont su cantonner leur relation avec les membres dominants de l'aristocratie à des rapports de type personnel qu'ils sont parvenus à éviter de faire fonctionner ces liens de subordination comme base de la construction d'une principauté territoriale (c'est-à-dire comme liens de sujétion) - et rien ne le montre mieux que l'engagement de ces nobles dans la construction même des principautés: ses formes reprenaient celles, typiques de la féodalité, du lien personnel ${ }^{26}$. Il est en effet extrêmement frappant que les relations de service présentent exactement la même évolution que les relations de vassalité : elles sont caractérisées par une augmentation de leur nombre, permettant l'élargissement géographique des princes avec lesquels elles sont entretenues, c'est-à-dire la perte du monopole du prince local. Cet élargissement, comme pour les fiefs, ne veut pas dire que ce dernier disparaisse ; si, pour les fiefs, cela pourrait être considéré comme une rigidité liée à leur caractère héréditaire, le service montre que, en fait, le refus de briser les liens avec le prince local était un choix, et que l'engagement auprès de princes extérieurs n'était pas concurrent mais complémentaire : il s'agissait simplement de s'assurer ainsi un contre-poids permettant que les relations de subordination ne soient pas considérées comme

${ }^{26}$ - Précisons ce qu'il convient entendre par « lien personnel» : non pas lien intersubjectif, comme le montre le caractère général des lettres de fief («alles zu Thun, das ein getrewer Lehenman seinem Lehenherrn zu Thunde schuldig und pflichtig ist »; il n'est nullement question de la relation entre deux individus, mais entre deux positions : Lehenmann, Lehenherr, pouvant être occupées par n'importe quel individu sans que la relation ne soit en rien transformée), mais simplement lien non basé sur une appartenance spatiale. 
une sujétion, ce qui autorisait ainsi à les conserver, avec tous les avantages qu'elles apportaient ${ }^{27}$; il s'agissait non de rompre avec le prince local, mais d'aménager ces relations au profit des nobles Il faut en conclure que l'apparition de nouvelles formes de relations entre membres dominants et dominés de l'aristocratie à la fin du Moyen Âge, loin de marquer le passage à un monde dé-féodalisé, ne fut que le moyen du renforcement des relations féodales grâce à leur redoublement par d'autres rapports, d'un type nouveau ; en effet, au-delà de leur apparente dissimilitude concrète, les deux types de relation relèvent d'une même logique. Leur complémentarité est particulièrement visible en ce qui concerne les rapports entre les Thüngen et les margraves de Brandebourg : les deux parties entrent d'abord en contact par le biais du service gagé, puis nouent des liens féodo-vassaliques (sans que les relations de service disparaissent pour autant). Cependant, cette complémentarité des relations de vassalité et de service ne vaut pas pour les seuls nobles (que favorise le caractère personnel des deux types de relations) : elle opère tout autant pour les princes. «Comme les nobles sont de plus en plus intéressés par les fiefs pour des raisons successorales, s'est établi une sorte d'échange tacite à partir du début $d u \mathrm{Xv}^{\mathrm{e}}$ siècle (ce qui correspond à l'apparition des Mannlehen à transmission collatérale) : d'abord le service contractuel, ensuite le fief. Bref, la relation de causalité ancienne "service, donc fief" serait ainsi recréée non plus au sein de la relation féodo-vassalique elle-même, mais par l'imbrication de celle-ci dans un système plus large de relations entre seigneurs et chevaliers ${ }^{28}$. »

La seule relation qui, nous semble-t-il, sorte vraiment du cadre féodo-vassalique est celle que Joseph Morsel appelle le « service financier », ce par quoi il désigne (en renversant, par cette désignation, le sens usuellement donné à cette pratique) les mort-gages princiers, d'une importance exceptionnelle dans l'Allemagne tardo-médiévale - surtout si l'on considère que la justification de l'établissement d'une imposition princière y a souvent été non pas, comme en France et Angleterre, la guerre, mais l'endettement princier. Or les débiteurs princiers des Thüngen présentent une structure radicalement différente de celle que l'on peut observer dans les autres types de relations des Thüngen avec les princes, puisque l'essentiel des crédits accordés l'ont été aux seuls évêques de Wurtzbourg, c'est-à-dire au prince local ${ }^{29}$. Comme la

27 - Possession de fiefs, rémunération dans le cas des « retenues » militaires ou de l'exercice d'offices, qui permettent également l'exercice d'un pouvoir délégué.

28 - J. Morsel, La noblesse contre le prince..., op. cit.,p. 00.

29 - Nous nous démarquons donc de Joseph Morsel sur ce point : si les relations de prêt sont en apparence «personnelles» au même titre que celles d'ordre féodo-vassaliques et de service, elles sont de facto territorialisées. Le mode concret de réalisation de ce type de 
position socio-politique relative du créancier et du débiteur est donc fortement hiérarchisée au détriment des Thüngen, c'est, ainsi que le montre J. Morsel, le créancier qui dépend de son débiteur, aussi bien parce qu'il n'est pas capable d'imposer le remboursement (le conflit militaire du second quart $\mathrm{du} \mathrm{Xv}^{\mathrm{e}}$ siècle, qui avait pour origine les dettes non remboursées de l'évêque, se termina aux dépens des Thüngen, qui durent renoncer à leurs créances), que parce qu'il est à la merci, dans son contrôle des biens à lui engagés, d'un désengagement que le débiteur peut opérer à tout moment (les difficultés financières n'entrant pas ici en ligne de compte, puisqu'il ne manque pas d'exemples où les évêques engagent tel bien auprès de tel noble pour désengager tel autre bien auprès de tel autre noble) ${ }^{30}$. Le créancier noble est ainsi doublement attaché à son débiteur princier : parce qu'il lui faut soutenir ses entreprises (notamment celles visant à l'établissement d'un impôt) pour espérer pouvoir être remboursé, et parce que seules ses bonnes relations avec le prince pourront le convaincre de ne pas désengager des biens dont le noble désire garder le contrôle. Ainsi, l'endettement colossal des évêques de Wurtzbourg (qui alla jusqu'à préoccuper le concile de Bâle), loin d'être la marque de la faillite de cette principauté ecclésiastique naissante, était pour elle le moyen d'intégrer fermement les nobles sur une base territoriale, ce dont n'étaient capables ni les relations féodo-vassaliques ni les relations de service. Cette intégration passait certes par l'abandon du contrôle de certains biens, mais un abandon qui, au contraire de celui découlant de l'inféodation, rendait à tout moment possible la reprise en main (par remboursement du créancier). On ne s'expliquerait d'ailleurs pas, si les raisons de l'endettement princier avaient été exclusivement financières, que cet endettement n'ait été opéré qu'auprès de nobles (alors que des institutions ecclésiastiques de moindre envergure que l'évêché recouraient

relations a ainsi inversé le sens que paraissait devoir leur conférer leur forme. Il convient d'ailleurs de noter que, si les fiefs et le service du prince ont renforcé l'autonomie des nobles, ce n'est pas en raison de leur caractère qui serait par essence personnel, mais parce que les nobles ont su faire de l'aspect personnel de ces types de relations leur caractéristique principale.

30 - L'argumentation pourrait paraître contradictoire qui, d'un côté, affirme que les nobles ne peuvent se faire rembourser et, inversement, prétend qu'ils préféraient n'en rien faire. Mais les opérations de crédit au prince ne sont pas homogènes : si certaines permettent, dans la zone d'implantation du lignage, d'acquérir des gages (que l'on entend conserver), pour d'autres opérations les gages n'ont, en raison de leur localisation périphérique par rapport aux intérêts du lignage, d'autre intérêt que la garantie qu'ils apportent (limitée, puisqu'elle ne porte que sur la rémunération du crédit, pas sur son remboursement, et que le créancier ne peut vendre son gage qu'avec l'accord du débiteur). 
massivement, au XIV ${ }^{\mathrm{e}}$ siècle, au crédit du patriciat urbain et des juifs), ce qui compliquait (et donc renchérissait) les circuits de crédit, puisque bien souvent ces nobles devaient eux-mêmes s'endetter pour pouvoir rassembler les sommes que le prince réclamait qu'ils lui prêtassent : l'endettement princier ne concernait que les nobles parce que c'étaient eux seuls qu'il importait, par ce biais, de contrôler ${ }^{31}$. Ainsi l'endettement permettait-il doublement la mise en place d'une principauté territoriale : par l'imposition frappant les roturiers (justifiée par la nécessité de réduire l'endettement), et par la dépendance nobiliaire créée par la relation de crédit; système parfait, puisque la mise en dépendance des deux groupes s'entretenait réciproquement (les villes ne pouvaient refuser l'impôt, faute de voir l'évêque devenir encore plus dépendant de ses débiteurs nobles, et donc s'accroître, à leur détriment, l'influence de ces derniers ; et la nécessité de se faire rembourser incitait les nobles à soutenir l'évêque dans ses tentatives pour institutionnaliser l'impôt). Il me semble donc nécessaire d'aller plus loin encore que ne le fait J. Morsel dans le renversement des perspectives : les relations de crédit ne sont pas un «service financier $»^{32}$, désignation qui les apparente aux autres types de liens personnels (renforçant les lignages, et indirectement la noblesse), mais le moyen de 1 '« enrégimentement territorial» (ce qui ne veut pas dire que les nobles ne tirent pas profit de ces crédits, mais qu'ils y perdent leur non-sujétion au prince).

L'échec de la construction étatique, au moins dans l'évêché de Wurtzbourg, paraît donc directement lié à l'abandon de l'endettement princier au milieu du $\mathrm{Xv}^{\mathrm{e}}$ siècle, et il est frappant que cet abandon soit concomitant du renforcement de la position, face à l'évêque, du chapitre cathédral (exclusivement composé de nobles), renforcement qui s'exprime notamment par l'obligation nouvelle pour l'évêque de prêter serment aux capitulations électorales (pour la première fois en 1444), par lesquelles il s'engage à tout faire pour réduire l'endettement de son Église. De là une réorganisation complète des relations entre l'appareil princier naissant et les nobles, ceux-ci y assurant désormais des fonctions non plus par le biais des châtellenies qui leur étaient engagées, mais en raison de contrats qui en font des officiers gagés - forme apparemment adaptée à la «modernité » de l'État, mais qui, en fait, signait l'échec de son établissement. Aussi bien - ceci pour dégager de la démonstration de Joseph Morsel ses

31 - Et, inversement, cette concentration de l'endettement princier sur les seuls nobles rendait pour ces derniers leur soutien au prince d'autant plus inévitable que du prince dépendait leur capacité de rembourser leurs propres créanciers (ou, dans le cas des juifs, de les déposséder de leurs créances).

32 - Encore moins un «crédit féodal», selon l'expression de Rolf Sprandel, quoique ce fussent des vassaux qui le consentaient. 
implications abstraites - une genèse ne consiste-t-elle jamais en l'institutionnalisation au sein d'une structure autre, par additions successives, des éléments destinés à former une structure nouvelle (car, ainsi que le montre l'exemple des officiers franconiens de la fin du Moyen Âge, tant que ces éléments nouveaux ne forment pas une structure, c'est toujours dans le sens d'un renforcement de celle au sein de laquelle ils apparaissent qu'ils fonctionnent), mais en l'utilisation transformée d'éléments de la structure qu'il s'agit d'éliminer ; une genèse n'est jamais, rapportée à son aboutissement, qu'un apparent paradoxe, dont le mode opératoire n'est pas la novation mais la perversion ${ }^{33}$.

Parler d'échec de la construction princière, c'est aussi poser la question des formes de pouvoir apparues en lieu et place de cet État que l'on trouve ailleurs en Europe ; c'est donc poser la question des modes par lesquels les nobles non titrés ne sont pas seulement parvenus à éviter leur assujettissement, mais ont construit leur pouvoir (les deux éléments ne pouvant évidemment être dissociés) - si l'on veut bien admettre que, contrairement à leur discours, il s'agissait bel et bien d'une construction, et non de la préservation de coutumes immémoriales.

\section{Invention de la noblesse et autonomisation de la noblesse}

Le problème décisif consistait à passer d'une structuration en lignages juxtaposés, qui étaient le vecteur de l'assignation à leurs membres d'une identité de groupe plus vaste mais secondaire (puisque découlant de l'appartenance au lignage), et n'aboutissant qu'à un agrégat de forme additive (c'est-à-dire n'ayant pas de réalité autre que celle de ses parties, et ne pouvant de ce fait devenir l'objet d'une conscience), à une structuration en un groupe dont les membres tirent leur identité (un groupe donc préalable à l'existence de ses parties), une identité spécifiée comme appartenance à un lignage (cette appartenance devenant donc secondaire) : toute la difficulté était de passer d'une structuration où l'on appartenait à un lignage, ce qui signifiait que l'on était noble, à une autre où l'on appartenait à la noblesse, et donc à un lignage. Une telle transformation requérait d'être soutenue par un ensemble de pratiques pas seulement discursives.

La première de ces pratiques permettant de faire exister le groupe noble comme une entité qui ne fût pas seulement agrégée est l'alliance matrimoniale, dont on a déjà traité mais qu'il convient désormais d'envisager sous un autre angle : celui du recrutement des conjoints. Deux éléments, à suivre l'exemple des Thüngen, le caractérisent : d'une part, son extension

\footnotetext{
33 - Ce que l'on voit aussi bien dans le fait que l'autonomie de la noblesse vis-à-vis des princes a été construite par le biais des relations féodo-vassaliques et de service.
} 
géographique progressive à l'ensemble de la Franconie, d'autre part, la division des lignages avec lesquels sont contractés des mariages en deux groupes représentant chacun la moitié des unions (d'une part $25 \%$ des lignages affins avec chacun desquels a été scellée plus d'une alliance ; d'autre part $75 \%$ des lignages avec chacun desquels les Thüngen ne se sont alliés qu'une fois). Ces deux caractéristiques sont liées, de façon complexe. Lorsque plusieurs unions ont eu lieu avec un même lignage, elles ont toujours été homogamiques, tandis que bon nombre des unions sans suite étaient hypogamiques; par ailleurs, les lignages avec lesquels les alliances se répétaient étaient proportionnellement plus éloignés dans l'espace, et plus la distance géographique était grande, plus la concentration des mariages sur les lignages ayant fourni plusieurs conjoints était importante. Ainsi, l'un des deux groupes d'alliés permettait de combler la distance sociale entre les nobles, tandis que l'autre avait pour fonction de pallier la distance géographique. Les alliances se divisaient donc en deux ensembles : d'un côté, celles, homogames et répétées, qui servaient à entretenir un réseau matrimonial, socialement unifié mais géographiquement différencié ; de l'autre, les unions hypogames locales, utilisées pour créer des liens matrimoniaux avec des lignages aussi nombreux que possibles mais avec le plus faible nombre possible de mariages. La raison de cette bipartition semble claire : la multiplication des alliances n'est intéressante qu'avec des lignages de même rang, et, par ailleurs, seule cette multiplication permet de pallier les effets de l'absence d'interactions concrètes fréquentes, impliquée par l'éloignement géographique ; l'alliance est le seul moyen d'entretenir un réseau à distance (tandis qu'à proximité elle ne fait que renforcer, ponctuellement, un réseau fondé sur d'autres types d'interactions, liées à la gestion seigneuriale ${ }^{34}$ ), mais comme l'entretien de ce réseau nécessite le renouvellement fréquent des alliances, l'on ne s'engage qu'avec des lignages de même rang, afin que l'investissement humain (en nombre de membres du lignage) soit en rapport avec le bénéfice social. Il convient toutefois d'aller plus loin que ne le fait Joseph Morsel, en ne se limitant pas à la considération des alliances d'un seul lignage puisque, si l'on fait l'hypothèse que les alliances des autres lignages fonctionnent selon le modèle dégagé par lui pour les Thüngen, il apparait alors que l'ensemble des alliances forme structure. En effet, si l'on se rappelle que la Freundschaft est largement fondée sur l'affinité, alors les mariages à distance avec un petit nombre de lignages de même rang introduisent dans le réseau local de la parenté de ces lignages, réseau rendu aussi vaste que possible par la non-concentration des alliances sur certains lignages (avec pour conséquence le caractère hypogamique de nombreuses alliances,

34 - Ces alliances visent donc à assurer la cohésion d'un groupe dominant localisé, et leur logique est à chercher dans le rapport aux dominés. 
bien que le caractère hypogamique de ces alliances passe au second plan dans la mesure où leur logique n'est pas l'intégration dans un réseau nobiliaire, à l'échelle régionale, mais dans un réseau seigneurial, à l'échelle locale). Si certains des lignages locaux auxquels on s'allie sont, à l'échelle régionale, inférieurs socialement dans la mesure où leurs possessions ne s'étendent pas à plusieurs zones de Franconie, en revanche, à l'échelle locale, leurs possessions, pour être concentrées dans cette seule zone, y représentent un facteur non dénué d'importance : les Thüngen jugeaient donc utile de s'allier avec eux, s'ils avaient eux-mêmes des possessions dans cette zone. La double logique spatiale/sociale du système d'alliance est donc structurellement assurée par le fait que les alliances hypogamiques locales ne sont pas perçues comme telles : les lignages ne les contractent pas parce qu'elles seraient nécessaires au bon fonctionnement de la structure et qu'elles seraient, ainsi, indirectement profitables au lignage, mais parce que, directement, dans le cadre d'une logique seigneuriale localisée, elles sont nécessaires et profitables au lignage. Les alliances contractées ont donc une double finalité : une implantation locale, dont l'utilité est de rendre attractif pour des lignages éloignés le mariage dans le lignage ; tandis que, inversement, cet intérêt même de celui-ci pour ceux-là lui permet de s'intégrer, par leur biais, aux réseaux localisés de leur parenté cognatique dans des zones distantes au sein desquelles le lignage considéré ne serait autrement pas capable de s'implanter.

On voit alors que l'alliance permet aux nobles leur intégration sociale (par delà les différences de fortune et de pouvoir, sans nullement viser, toutefois, à éliminer ces différences, au contraire exprimées par la double structure de l'alliance) et géographique. Cette fonction de l'alliance n'est en rien un donné, puisqu'elle n'a été rendue possible que par la transformation du système successoral : en effet, à partir du moment où s'imposait la succession agnatique, l'alliance ne pouvait plus être pour un lignage le moyen de se créer des droits sur le patrimoine d'autres lignages, ni, par conséquent, d'élargir sa seigneurie. Si le mariage « successoral », parce que sa fonction était seigneuriale, était nécessairement à la fois concentré géographiquement et homogamique ou hypergamique, puisqu'il n'était intéressant ni d'acquérir des possessions éloignées, qu'il eût été impossible de contrôler, ni de se marier dans un lignage pauvre de la succession duquel il y avait peu à attendre, la fin de la fonction successorale de l'alliance autorise l'inversion de ces caractères : non seulement l'aire de recrutement des conjoints s'élargit, mais le recrutement n'est plus homogamique qu'avec les lignages les plus éloignés. L'alliance n'a plus pour fonction la reproduction (élargie) du lignage (par captation d'héritages), mais la reproduction de la noblesse en tant que groupe unifié. 
On en est donc revenu à la Freundschaft comme principe essentiel de l'organisation du groupe noble. L'alliance matrimoniale, aussi efficacement organisée fût-elle, ne permettait toutefois pas d'étendre la Freundschaft d'un lignage à l'ensemble des lignages, ne serait-ce que par l'inévitable limitation du nombre de parents disponibles pour contracter des alliances $^{35}$. Les liens entre nobles devaient donc passer également par les voies de l'alliance non matrimoniale, l'Einung (ligue), dont les principes étaient exactement ceux de la Freundschaft (qui, rappelons-le, n'était pas exclusivement parentélaire) : aide contre les non-membres du groupe et arbitrage interne ; ainsi, si leurs mariages ont mis les Thüngen en relation directe avec une cinquantaine de lignages, les différentes ligues anti-princières auxquelles ils participèrent à partir de 1402 les ont rapprochés de cent quarante lignages (parmi lesquels les cinquante lignages alliés). [Saut de $\S]$

Un second type de lien institutionnalisé entre nobles autre que l'alliance est représenté par les co-châtellenies non lignagères, d'une grande importance symbolique puisqu'elles portent sur cette pratique même qui, à partir du deuxième tiers du $\mathrm{XIV}^{\mathrm{e}}$ siècle, avait permis de renforcer la conscience lignagère par l'institution d'indivisions successorales agnatiques sur les moyens les plus importants et les plus représentatifs du pouvoir nobiliaire, à un moment où la mutation du droit des fiefs n'avait pas encore eu lieu. Les co-châtellenies qui apparaissent au tournant $\mathrm{du} \mathrm{XIV}^{\mathrm{e}}$ siècle (c'est-à-dire au moment de la réorganisation du droit des fiefs), au contraire, ne mettent comme critère à leur appartenance que la qualité de noble, et n'établissent pas de privilège successoral agnatique, ce qui contribue à faire circuler les parts de ces indivisions de lignage en lignage. Une telle attitude s'explique par la localisation différentielle des châteaux faisant l'objet d'indivisions lignagères et non lignagères : tandis que les premiers sont situés dans la zone où se concentrent les possessions d'un lignage, les seconds lui sont toujours extérieurs. [Suppression du saut de §] La sortie de l'indivision non lignagère n'a donc, pour le lignage, pas de conséquence militaire, ce qui permet d'assigner à la circulation des parts une fonction de lien social entre les lignages. Ce qui vaut pour les

\footnotetext{
35 - Limitation partiellement contournée toutefois par le fait que la stratégie de reproduction sociale des nobles non titrés, contrairement à celle de leurs pairs titrés, n'était pas fondée sur la limitation du nombre des membres du lignage, non-limitation rendue possible par le principe successoral agnatique (c'est-à-dire par le fief nouvelle manière), qui était donc la condition de possibilité de l'unification des nobles par l'alliance matrimoniale. Ainsi, principe agnatique (représenté par le lignage et le fief) et principe cognatique (représenté par l'importance donnée à l'alliance) non seulement ne sont pas contradictoires mais, au contraire, sont impensables l'un sans l'autre.
} 
Thüngen étant sans doute également vrai pour les autres lignages qui fournissent des coparsonniers, la fonction des co-châtellenies non lignagères n'est donc vraisemblablement pas, comme le veut J. Morsel, d'assurer l'intégration dans un réseau local éloigné (ce qui est la fonction des alliances matrimoniales homogamiques), mais de provoquer de façon relativement stochastique une alliance d'intérêts entre des lignages tous identiquement éloignés du château dont ils sont coparsonniers, manière de signifier que la noblesse est un groupe substantiel, au sein duquel les interactions ne sont pas dues seulement au choix (matrimonial, ou politique avec les ligues) et à la localisation, mais aussi au simple hasard, réunissant des semblables qui, en tant que semblables, ont vocation à être réunis ${ }^{36}$.

L'unité concrète des nobles a donc été assurée par un ensemble de pratiques portant sur des objets fondamentaux (l'alliance matrimoniale, l'alliance politique, le contrôle des châteaux) grâce à la réorganisation de pratiques antérieures (cas du mariage et des cochâtellenies) ou à la création de nouveaux modes d'interaction (cas des ligues). Toutefois, tant le bon fonctionnement (la reproduction automatique) de ces pratiques unifiantes que leur création (dans un monde qui fait de la coutume une valeur, particulièrement chez les nobles qui se réclament de leur Herkommen und Freiheit, «origine / tradition et liberté ») ne peuvent s'opérer que si ces pratiques apparaissent à ceux qui les portent comme naturelles, et donc seulement si un discours ayant acculturé l'idée de « la noblesse » comme groupe substantiel leur est préexistant. De cela, il n'est point de meilleur exemple que la Ritterschaft, nom que prend l'organisation anti-princière des nobles à partir de la fin $\mathrm{du} \mathrm{xv}^{\mathrm{e}}$ siècle, moment où les ligues, jusque-là toujours conçues comme limitées dans le temps, commencent à s'inscrire et dans la durée et dans l'espace (en se divisant en régions, chacune dirigée par un collège exécutif). Ce nom, en effet, justification de l'entreprise à lui seul, ne peut s'entendre si l'on ne considère l'extraordinaire fortune de l'idéologie chevaleresque à partir du milieu du xv ${ }^{\mathrm{e}}$ siècle. Cette fortune nouvelle (ou plutôt : renouvelée) se traduit aussi bien dans l'anthroponymie (un tiers des hommes nobles portant désormais le nom d'un saint chevalier, alors que ces prénoms étaient encore des hapax à la fin du XIV ${ }^{\mathrm{e}}$ siècle) que par l'organisation, entre 1479 et 1487, de grands tournois rassemblant la noblesse de toute la moitié sud de l'espace allemand ${ }^{37}$. Or ce référent chevaleresque, loin d'être une anachronique nostalgie à la Huizinga, représentait le

\footnotetext{
${ }^{36}$ - Cet accent mis sur le hasard me paraît bien rendre compte du fait que « les autres cohéritiers sont largement étrangers au cercle des fréquentations régulières des Thüngen : les co-châtellenies sont des groupements de nobles qui viennent en complément des réseaux matrimonial et associatif»(J. Morsel, La noblesse contre le prince..., op. cit., p. 00).
} 
moyen d'articuler un discours offensif quant à la place des nobles dans la société et leur organisation interne ; en effet, le problème étant celui de la définition des personnes admises à tournoyer, l'exigence de quatre aïeuls nobles, d'un côté, revenait à insister sur l'alliance comme fondement de la noblesse ${ }^{38}$, et, de l'autre, permettait d'exclure les bourgeois (ce d'autant plus clairement que les joutes se déroulaient justement dans l'espace urbain) ${ }^{39}$. «Dans le tournoi, l'aristocratie met en scène un code de valeurs qu'elle pose comme communes à tous ses membres »(p.00). Ce respect des valeurs, qui donne son prix au groupe, lui permet de justifier, face aux princes, le refus de la sujétion, transférant ainsi le terme Ritterschaft d'une pratique symbolique de sociabilité à une organisation politique antiterritoriale.

Mais l'apport essentiel de J. Morsel quant à la fonction socialement constructrice des discours historiques (apport déconstructeur des discours historiographiques) tient dans la démonstration de l'émergence de la notion de noblesse (en tant que groupe) au cours du deuxième tiers du $\mathrm{Xv}^{\mathrm{e}}$ siècle ${ }^{40}$. De même que la lexicométrie (démarche empirique s'il en est !) lui a permis, en dégageant l'importance centrale de la Freundschaft, de briser le mythe d'une structuration fondamentalement agnatique de la parenté nobiliaire, de même l'étude quantifiée d'un autre type de vocabulaire lui permet de cerner une évolution des représentations qui rassemble toutes les observations précédentes, et le rend donc capable d'expliquer la genèse et la reproduction des discours et pratiques que l'on a détaillés. En effet, jusque dans les

${ }^{37}$ - ID., « Le tournoi, mode d'éducation politique en Allemagne à la fin du Moyen Âge », in Éducation, apprentissages, initiation au Moyen Âge, Montpellier, Les Cahiers du CRISIMA, 1, 1993, pp. 309-331.

38 - Sans pour autant nier l'importance de la dimension lignagère : si l'appartenance à un lignage ne fait pas le noble, en revanche l'alliance de quatre lignages vaut preuve de noblesse. La différence n'en reste pas moins écrasante par rapport au système des quartiers de noblesse, qui exclut radicalement l'alliance et - est-ce vraiment un hasard ? - a été celui adopté dans une zone à «État» comme la France.

39 - Comme au $\mathrm{Xv}^{\mathrm{e}}$ siècle il n'était pas exceptionnel que des patriciens se fissent adouber (parfois par l'empereur lui-même, et à Rome), cette exclusion n'avait rien d'évident puisqu'il s'agissait d'interdire à des chevaliers la participation au tournoi, alors même qu'elle était autorisée à de simples écuyers - mais nobles.

40 - ID., « Die Erfindung des Adels. Zur Soziogenese des Adels am Ende des Mittelalters das Beispiel Frankens », in O. G. Oexle et W. Paravicin (dir.), Nobilitas: Funktion und Repräsentation des Adels in Alteuropa, Göttingen, Vandenhoeck \& Ruprecht, 1997, pp. 312-375. 
années 1440, le champ sémantique de la noblesse n'apparaît pour l'essentiel que sous une forme adjectivée (edel), le substantif (Adel), plus rare, ne servant qu'à désigner la qualité de l'individu auquel est appliqué l'adjectif; ensuite, au contraire, le substantif domine et, si le terme ne change pas, son sens est en revanche complètement modifié, puisqu'il renvoie désormais à un groupe. Le noble, alors, ne l'est plus parce qu'il incarne une qualité, mais parce qu'il appartient au groupe noble (qui n'est plus le groupe des nobles) : ce n'est plus de lui-même, et de son lignage, qu'il tire son identité noble, mais de l'existence de ce groupe, dont dépend la sienne : désormais, il n'est plus ein edler Mann ( « un homme noble ») mais einer vom Adel (« un de la noblesse »). Le substantif Adel n'est plus une qualité s'incarnant à volonté dans des individus, mais désigne désormais un groupe qui est investi de cette qualité. Le noble ne peut plus exister sans la noblesse (comme groupe), tandis que celle-ci existe indépendamment de lui.

C'est sans doute pour ne s'être pas donné les moyens (empiriques) de déconstruire la catégorie de «noblesse » comme groupe (parce que cette catégorie, pour pleinement moderne qu'elle fût, paraissait fallacieusement ne relever que du passé), que les historiens travaillant sur la fin du Moyen Âge allemand n'ont pu apercevoir que cette période avait connu une restructuration sociale d'une ampleur considérable, mais se situant ailleurs que là où l'on prétendait la voir (modernité « bourgeoise », proto-capitalisme, etc.). Tout le mérite de Joseph Morsel est d'avoir attiré l'attention non seulement sur l'objet véritable de la mutation (la création de la noblesse comme groupe), mais aussi sur les mécanismes de cette dernière, qu'il a su mettre à nu : de ne pas s'être contenté de voir le problème, mais de l'avoir résolu - ce qui impliquait de rendre compte dans le même mouvement de l'échec de la constitution des principautés territoriales. Ce faisant, son œuvre représente un approfondissement décisif de la réflexion sur la « genèse de l'État moderne », puisque la constitution de la noblesse s'est aussi réalisée là où cette genèse a été couronnée de succès - mais, ici (du moins si l'on suit le cas de la France), c'est l'État lui-même qui a créé la noblesse comme groupe. Nos remarques n'avaient pour objectif que de poursuivre l'enquête dans les voies tracées par Joseph Morsel ; et si désormais nous pouvons emprunter ces pistes, ce n'est que parce qu'il est parvenu à les ouvrir.

Julien Demade CNRS (Laboratoire de médiévistique occidentale de Paris) 